\title{
RISE IN INCIDENCE OF DIFFERENTIATED THYROID CANCER IN THE SOUTH OF SPAIN
}

${ }^{1}$ Carral F, ${ }^{2}$ Ruiz G, ${ }^{2}$ Tinoco R, ${ }^{3}$ González-Outón J, ${ }^{4}$ Vega V, ${ }^{4}$ Robles M, ${ }^{1}$ Jiménez Al, ${ }^{1}$ García C, ${ }^{1}$ Ayala C.

1 Endocrinology, ${ }^{2}$ Internal Medicine, ${ }^{3}$ Clinical Information, ${ }^{4}$ Surgery Departments. Puerto Real University Hospital, Cádiz, Spain.

Background: Recent studies have shown that the incidence of differentiated thyroid cancer (DTC) is increasing.

Objective: Evaluate the incidence of DCT in a center of the south of Spain for the last 15 years (2000-2014) and analyze the clinical and morphological factors related to DTC.

Methods: We reviewed the series of patients undergoing thyroid surgery at our Hospital over a 15-year period and identified patients with DTC. Patients were divided in three periods: 2000-2004, 2005-2009 and 2010-2014 and incidence rates were calculated. The variables gathered were age, sex, date of diagnosis, clinical presentation, hormonal status and cytology, size, morphology and extension of the tumor.

Results: Results are expressed in tables 1 and 2 and in graphic 1.

Table 1. DTC incidence in analyzed periods and annual percentage change

\begin{tabular}{l|c|c|c|c}
\multicolumn{1}{c|}{ Period } & $\begin{array}{c}2000-2004 \\
(\mathrm{n}: 48)\end{array}$ & $\begin{array}{c}2005-2009 \\
(\mathrm{n}: 71)\end{array}$ & $\begin{array}{c}2010-2014 \\
(\mathrm{n}: 105)\end{array}$ & APC \\
\hline Total $^{1}(\mathrm{IC} 95 \%)$ & $3.69(3.67-3.70)$ & $4.84(4.82-4.85)$ & $6.82(6.81-6.83)$ & $5.20(5.19-5.21)$ \\
Men & $1.07(1.05-1.07)$ & $1.67(1.65-1.68)$ & $3.32(3.30-3.34)$ & $2.07(2.06-2.07)$ \\
Women & $6.35(6.33-6.37)$ & $7.90(7.88-7.92)$ & $10.17(10.14-10.19)$ & $8.26(8.25-8.27)$
\end{tabular}

${ }^{1}$ DTC cases /100.000 habitants/ year; APC: Annual percentage change.

Table 2. Characteristics of DTC in analyzed period

\begin{tabular}{|c|c|c|c|c|}
\hline Período & 2000-2004 & 2005-2009 & 2010-2014 & $\mathbf{P}$ \\
\hline Thyroidectomies (n) & 491 & 499 & 615 & - \\
\hline $\begin{array}{l}\text { DTC cases } \\
\text { Men (\%) } \\
\text { Women (\%) }\end{array}$ & $\begin{array}{c}48 \\
7(14.6 \%) \\
41(85.4 \%)\end{array}$ & $\begin{array}{c}71 \\
12(19.6 \%) \\
59(80.4 \%)\end{array}$ & $\begin{array}{c}105 \\
25(23.8 \%) \\
80(76.2 \%)\end{array}$ & 0.321 \\
\hline $\begin{array}{l}\text { Mean age (years) } \\
\text { Men } \\
\text { Women }\end{array}$ & $\begin{array}{l}45.4 \pm 13.6 \\
53.3 \pm 17.1 \\
44.1 \quad 12.8\end{array}$ & $\begin{array}{l}47.8 \pm 14.5 \\
47.9 \pm 14.7 \\
47.9 \quad 14.6\end{array}$ & $\begin{array}{l}45.9 \pm 15.0 \\
44.8 \pm 16.9 \\
46.2 \quad 14.5\end{array}$ & 0.595 \\
\hline Men/Women relation & 5.8 & 4.9 & 3.2 & 0.321 \\
\hline $\begin{array}{l}\text { Type of DTC } \\
\text { Papillary (\%) } \\
\text { Follicular (\%) }\end{array}$ & $\begin{array}{c}48 \\
43(89.6 \%) \\
5(10.4 \%)\end{array}$ & $\begin{array}{c}71 \\
67(94.3 \%) \\
4(5.7 \%)\end{array}$ & $\begin{array}{c}105 \\
93(88.6 \%) \\
12(11.4 \%)\end{array}$ & 0.416 \\
\hline Mean DTC size (mm) & $25.5 \pm 19.6$ & $15.6 \pm 11.4$ & $20.2 \pm 16.0$ & 0.007 \\
\hline Microcarcinomas (\%) & $10(20.8 \%)$ & $26(36.6 \%)$ & $30(28.6 \%)$ & 0.065 \\
\hline PTC non-MPTC/MPTC (\%) & 3.1 & 1.6 & 2.1 & 0.291 \\
\hline Thyroiditis (\%) & $10(20.8 \%)$ & $14(19.7 \%)$ & $29(27.6 \%)$ & 0.513 \\
\hline Multifocality (\%) & $16(33.3 \%)$ & $22(30.9 \%)$ & $39(37.1 \%)$ & 0.751 \\
\hline Bilaterality (\%) & $11(22.9 \%)$ & $17(23.9 \%)$ & $15(14.3 \%)$ & 0.171 \\
\hline Lymph node extension (\%) & $12(25.0 \%)$ & $17(23.9 \%)$ & $21(20.0 \%)$ & 0.581 \\
\hline Distance metastases (\%) & $1(2.1 \%)$ & $0(0.0 \%)$ & $5(4.7 \%)$ & 0.156 \\
\hline
\end{tabular}

Graphic 1. DTC in patients undergoing thyroid surgery in our Hospital during the period 2000-2014

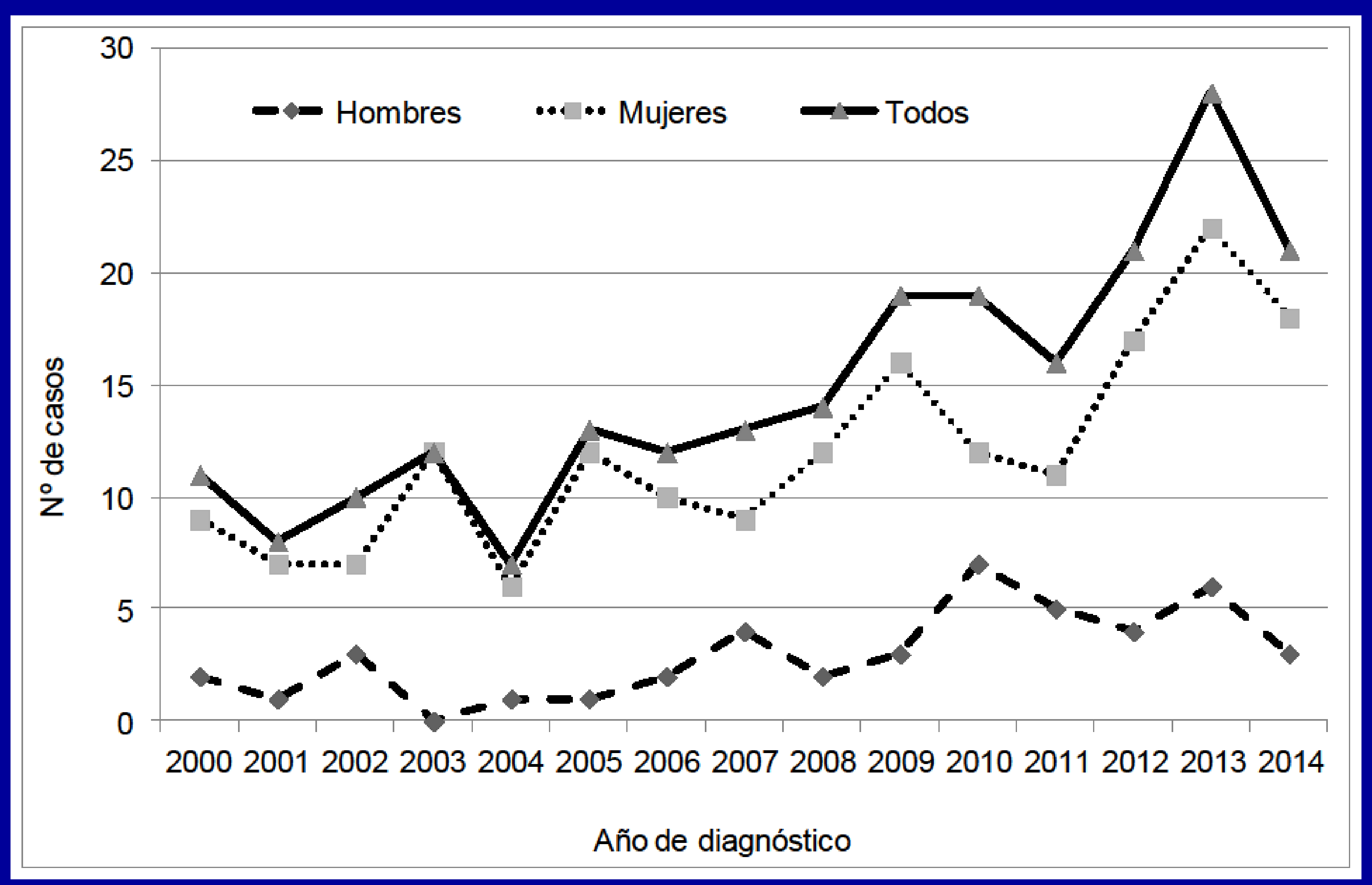

Conclusions: We report a similar rise in the incidence of DTC in the south of Spain to the overall reported in the international literature.

PTC: Pappillary hhyroid carcinoma; MPTC: Micropappillary hhyroid carcinoma.

${ }^{1} p<0,05$ in 2000-2004 and 2010-2014 periods vs 2005-2009 period. 